\title{
Surface Wave Echo in a Semi-Bounded Plasma
}

\author{
Hee J. Lee, Myoung-Jae Lee \\ Department of Physics, Hanyang University, Seoul, Korea \\ Email: hjlee@hanyang.ac.kr
}

Received 8 June 2016; accepted 5 August 2016; published 8 August 2016

Copyright (C) 2016 by authors and Scientific Research Publishing Inc.

This work is licensed under the Creative Commons Attribution International License (CC BY). http://creativecommons.org/licenses/by/4.0/

(c) (i) 0pen Access

\begin{abstract}
Plasma echo theory is revisited to apply it to a semi-bounded plasma. Spatial echoes associated with plasma surface wave propagating in a semi-bounded plasma are investigated by calculating the second order electric field produced by external charges and satisfying the boundary conditions at the interface. The boundary conditions are two-fold: the specular reflection condition and the electric boundary condition. The echo spots are determined in terms of the perpendicular coordinate to the interface and the parallel coordinate along which the wave propagates. This improves the earlier works in which only the perpendicular coordinate is determined. In contrast with the echo in an infinite medium, echoes in a bounded plasma can occur at various spots. The diversity of echo occurrence spots is due to the discontinuity of the electric field at the interface that satisfies the specular reflection boundary condition. Physically, the diversity appears to be owing to the reflections of the waves from the interface.
\end{abstract}

\section{Keywords}

Plasma Echo, Semi-Bounded Plasma, Boundary Condition

\section{Introduction}

Plasma echoes in an infinite plasma have long been known theoretically [1] [2] as well as experimentally [3]. Spatial echoes were theoretically investigated in a static situation where the non-propagating electric field is directed perpendicular to the interface of a semi-bounded plasma [4] [5]. If the perpendicular direction is designated as the $x$ direction, the electric field $E$ as well as the distribution function $f$ is spatially onedimensional: $E=E(x, t)$ and $f=f(x, v, t)$, where $x>0 \quad(x<0)$ is the plasma (vacuum) region. In this case, the corresponding Vlasov equation takes the form of a first order differential equation, and can be solved by satisfying the specular reflection boundary condition at the interface $x=0: f(v, 0)=f(-v, 0)$ [6]. This 
differential equation approach with the specular reflection boundary condition for a semi-bounded plasma has been shown to be entirely equivalent with the Fourier transform (with respect to $x$ ) under the recipe that the $E(x)$ is extended into the region $x<0$ in an odd function manner, $E(x)=-E(-x)$ [5]. This odd function extension of $E(x)$ gives rise to a surface term in the Fourier transform of the Poisson equation, which plays a significant role in the determination of the echo spots. It appears that this surface term, which the earlier authors entirely neglected, gives rise to diversity of echo spots [5]. Physically, the surface term manifests the reflection of the electric field at the boundary.

The echo phenomena is the result of a quadratic interaction of the two primary waves launched by two external charges at different locations (spatial echoes) or different times (temporal echoes). In response to the external charges, the plasma distribution function $f(x, v, t)$ is modulated with the exponential phase $\mathrm{e}^{i k(x-v t)}$, which is derived from the singularity at $\omega=k v$ of the linear response function. This term is called the free streaming term since $x=v t$ is the characteristic line of the Vlasov equation for a free particle. This rapidly modulating exponential phase makes the $f(x, v, t)$ more and more oscillatory as $t$ or $x$ increases, and consequently, $\int \mathrm{f} d v$ will become vanishingly small due to almost complete phase mixing. Therefore, in the first order, the phase mixing obliterates any appreciable effect on the macroscopic variable such as density perturbation. However, the second order distribution function which is a product of two first order distribution functions is not phase-mixed when or where the condition for a constructive interference is met, thereby the second order electric field does not vanish, resulting in an echo. It is evident from the expression for the product of two free-streaming exponentials $\mathrm{e}^{i k_{1}\left(x_{1}-v t_{1}\right)} \mathrm{e}^{i k_{2}\left(x_{2}-v t_{2}\right)}$ that a constructive interference can result in at a certain time (temporal echo) or a certain spot (spatial echo) such that $k_{1} x_{1}+k_{2} x_{2}=v\left(k_{1} t_{1}+k_{2} t_{2}\right)$.

In this work, we investigate spatial echoes in a semi-bounded plasma, taking a full account of the boundary terms which originate from the oddly continuation of the electric field. This work is an extension of the earlier paper by Lee and Lee [5]; the distribution function and the electric field are now spatially two-dimensional, allowing for the $z$-dependance. Therefore, the echoes are associated with the surface wave which is propagating in the $z$-direction. The second order electric field endowed with the additional $z$-dependance can be Fourierinverted by contour integration with unstraightforward analytic exercise, and delineating the echo condition requires extra complexity. The important boundary term is the discontinuity of the perpendicular electric field at the interface that is necessary to have the specular reflection boundary condition satisfied [5]. The diversity of echo occurrence spots has been experimentally reported [7] and can be explained by this boundary term. The identification of the echo spot associated with surface wave appears to be useful in experimental point of view [7].

\section{Formulation of the Problem}

We consider a plasma consisting of electrons and stationary ions, the latter forming the uniform background. The plasma is assumed to occupy the half-space $x \geq 0$. The region $x<0$ is assumed to be a vacuum. The perturbed electron distribution function $f(\boldsymbol{r}, \boldsymbol{v}, t)$ and the electric field $E(\boldsymbol{r}, t)$ will depend on $x$ and $z-$ coordinates with the $y$ coordinate ignored since $y$ direction has a translational invariance. We have the nonlinear Vlasov equation and the Poisson equation to describe the electrostatic perturbation:

$$
\frac{\partial}{\partial t} f(\boldsymbol{v}, \boldsymbol{r}, t)+\boldsymbol{v} \cdot \frac{\partial f}{\partial \boldsymbol{r}}-\frac{e}{m} \boldsymbol{E}(\boldsymbol{r}, t) \cdot \frac{\partial f}{\partial \boldsymbol{v}}=0
$$

with $\boldsymbol{r}=\hat{\boldsymbol{x}} x+\hat{\mathbf{z}} z, \boldsymbol{v}=\hat{\boldsymbol{x}} v_{x}+\hat{\mathbf{z}} v_{z}, \boldsymbol{E}=\hat{\boldsymbol{x}} E_{x}+\hat{\mathbf{z}} E_{z}$

$$
\nabla \cdot \boldsymbol{E}=\frac{\partial E_{x}}{\partial x}+\frac{\partial E_{z}}{\partial z}=4 \pi\left(-e \int \mathrm{d}^{2} v f+\rho_{0}(x, t)\right)
$$

where $f$ is a two-dimensional distribution function, and $\rho_{0}$ represents the external charges:

$$
\rho_{0}(x, z, t)=\left(\rho_{1} \mathrm{e}^{i \omega_{1} t} \delta\left[k_{0}\left(x-L_{1}\right)\right] \delta\left[k_{0}\left(z-S_{1}\right)\right]+1 \rightarrow 2\right)
$$

$k_{0}$ is introduced to make the argument of the $\delta$-function dimensionless, and $1 \rightarrow 2$ means the replica of the preceding term with the subscript 1 replaced by subscript 2. We solve the simultaneous Equations (1) and (2) for a given $\rho_{0}(x, t)$ as prescribed by Equation (3). In mathematical terms, we have an inhomogeneous system, driven by the source term in Equation (3). The responses $f$ and $E$ should be determined by $\rho_{0}$. 
The kinetic equation is supplemented by the kinematic boundary condition which we assume to be the specular reflection condition

$$
f\left(v_{x}, v_{z}, x=0, z\right)=f\left(-v_{x}, v_{z}, x=0, z\right)
$$

This specular reflection boundary condition is automatically satisfied by extending the electric field component $E_{x}(x)$ in odd function manner into the region $x<0$, i.e., $E_{x}(-x)=-E_{x}(x)$. Assuming that the external perturbation is small, we solve Equations (1) and (2) by successive approximation. First, the linear solution of Equation (1) will be obtained for $f$ with the boundary condition (4). Substituting this solution in Equation (2) yields an integral equation for the electric field which is solved by Fourier transform. Then the linear solution will be used to obtain the higher order solutions. We work only up to the second order. The higher order distribution function should also satisfy the boundary condition (4). The electric field should satisfy the electric boundary conditions: the normal component of the electric displacement $D_{x}(x)$ and the tangential electric field $E_{z}$ are continuous across the interface. In this work, the Fourier transform is defined by

$$
\begin{gathered}
f(k, \omega)=\int_{-\infty}^{\infty} \mathrm{d} x \int_{-\infty}^{\infty} \mathrm{d} t f(x, t) \mathrm{e}^{-i k x+i \omega t} \\
f(x, t)=\int_{-\infty}^{\infty} \frac{\mathrm{d} k}{2 \pi} \int_{-\infty}^{\infty} \frac{\mathrm{d} \omega}{2 \pi} f(k, \omega) \mathrm{e}^{i k x-i \omega t}
\end{gathered}
$$

Let us Fourier transform Equations (1)-(3) with respect to $t$ and $z$ to write

$$
\begin{gathered}
-i(\omega-\boldsymbol{k} \cdot \boldsymbol{v}) f(\boldsymbol{v}, \boldsymbol{k}, \omega)-\frac{e}{m} \int \frac{\mathrm{d} \omega^{\prime}}{2 \pi} \int \frac{\mathrm{d}^{2} k^{\prime}}{(2 \pi)^{2}} \boldsymbol{E}\left(\boldsymbol{k}-\boldsymbol{k}^{\prime}, \omega-\omega^{\prime}\right) \cdot \frac{\partial}{\partial \boldsymbol{v}} f\left(\boldsymbol{v}, \boldsymbol{k}^{\prime}, \omega^{\prime}\right)=0 \\
i \boldsymbol{k} \cdot \boldsymbol{E}(\boldsymbol{k}, \omega)+N\left(k_{\mathrm{z}}, \omega\right)=4 \pi\left[-e \int f(\boldsymbol{k}, \boldsymbol{v}, \omega) \mathrm{d}^{2} v+\rho_{0}(\boldsymbol{k}, \omega)\right]
\end{gathered}
$$

where

$$
N\left(k_{z}, \omega\right)=E_{x}\left(0^{-}, k_{z}, \omega\right)-E_{x}\left(0^{+}, k_{z}, \omega\right)
$$

is derived from the discontinuity of $E_{x}$ at $x=0$. This N-term is characteristic of a semi-bounded plasma and responsible for the diversity of surface wave echoes, as compared with an infinite plasma. The external charges are Fourier transformed to

$$
\rho_{0}(\boldsymbol{k}, \omega)=\frac{2 \pi}{k_{0}^{2}}\left[\rho_{1} \delta\left(\omega+\omega_{1}\right) \mathrm{e}^{-i k_{x} L_{1}} \mathrm{e}^{-i k_{2} S_{1}}+1 \rightarrow 2\right]
$$

Equations (5) and (6) constitute a set of nonlinear simultaneous equations. We solve the set of equations by successive approximations in terms of perturbation series:

$$
\begin{gathered}
f(\boldsymbol{k}, \boldsymbol{v}, \omega)=f_{0}(\boldsymbol{v})+f^{(1)}(\boldsymbol{k}, \boldsymbol{v}, \omega)+f^{(2)}(\boldsymbol{k}, \boldsymbol{v}, \omega)+\cdots \\
\boldsymbol{E}(\boldsymbol{k}, \omega)=\boldsymbol{E}^{(1)}(\boldsymbol{k}, \omega)+\boldsymbol{E}^{(2)}(\boldsymbol{k}, \omega)+\cdots
\end{gathered}
$$

Breaking down Equations (5) and (6) order by order, we have

$$
\begin{gathered}
-i(\omega-\boldsymbol{k} \cdot \boldsymbol{v}) f^{(1)}(\boldsymbol{k}, \boldsymbol{v}, \omega)=\frac{e}{m} \boldsymbol{E}^{(1)}(\boldsymbol{k}, \omega) \cdot \frac{\mathrm{d} f_{0}}{\mathrm{~d} \boldsymbol{v}} \\
i \boldsymbol{k} \cdot \boldsymbol{E}^{(1)}(\boldsymbol{k}, \omega)+N\left(k_{z}, \omega\right)=4 \pi\left[-e \int f^{(1)}(\boldsymbol{k}, \boldsymbol{v}, \omega) \mathrm{d}^{2} v+\rho_{0}(\boldsymbol{k}, \omega)\right] \\
-i(\omega-\boldsymbol{k} \cdot \boldsymbol{v}) f^{(2)}(\boldsymbol{k}, \boldsymbol{v}, \omega) \\
=\frac{e}{m} \boldsymbol{E}^{(2)}(\boldsymbol{k}, \omega) \cdot \frac{\mathrm{d} f_{0}}{\mathrm{~d} \boldsymbol{v}}+\frac{e}{m} \int_{-\infty}^{\infty} \frac{\mathrm{d} \omega^{\prime}}{2 \pi} \int_{-\infty}^{\infty} \frac{\mathrm{d} k_{x}^{\prime}}{2 \pi} \int_{-\infty}^{\infty} \frac{\mathrm{d} k_{z}^{\prime}}{2 \pi} \boldsymbol{E}^{(1)}\left(\boldsymbol{k}-\boldsymbol{k}^{\prime}, \omega-\omega^{\prime}\right) \cdot \frac{\partial}{\partial \boldsymbol{v}} f^{(1)}\left(\boldsymbol{k}^{\prime}, \omega^{\prime}, \boldsymbol{v}\right) \\
i \boldsymbol{k} \cdot \boldsymbol{E}^{(2)}(\boldsymbol{k}, \omega)=-4 \pi e \int f^{(2)}(\boldsymbol{k}, \boldsymbol{v}, \omega) \mathrm{d}^{2} v
\end{gathered}
$$

The quantity $N\left(k_{z}, \omega\right)$ in Equation (9) should be determined in terms of the vacuum field from the electric field boundary condition: electric displacement $D_{x}(x)$ is continuous across the interface $x=0$, 


$$
D_{x}^{(1)}\left(0^{+}\right)=D_{x}^{(1)}\left(0^{-}\right)
$$

where $D_{x}^{(1)}\left(0^{-}\right)$equals to the vacuum electric field $E_{0}$.

\section{Linear Solution}

Equations (8) and (9), and $\nabla \times \boldsymbol{E}=0$ give

$$
\begin{gathered}
\boldsymbol{E}^{(1)}(\boldsymbol{k}, \omega)=\frac{i \boldsymbol{k}}{k^{2} \varepsilon(\boldsymbol{k}, \omega)}\left[N\left(k_{z}, \omega\right)-4 \pi \rho_{0}(\boldsymbol{k}, \omega)\right] \\
\varepsilon(\boldsymbol{k}, \omega)=1+\frac{\omega_{p}^{2}}{k^{2}} \int \mathrm{d}^{2} v \frac{\boldsymbol{k} \cdot \frac{\mathrm{d} f_{0}}{\mathrm{~d} \boldsymbol{v}}}{\omega-\boldsymbol{k} \cdot \boldsymbol{v}}
\end{gathered}
$$

is the dielectric function ( $\omega_{p}$ is the plasma frequency). $N$ is determined from the electric boundary condition as shown in the following. We need the normal component of electric displacement, $D_{x}(x)$ to enforce the boundary condition (12). By definition, $D_{x}(\boldsymbol{k}, \omega)=E_{x}(\boldsymbol{k}, \omega)+\frac{4 \pi i}{\omega} J_{x}(\boldsymbol{k}, \omega)$ where $J$ is the current:

$J_{x}(\boldsymbol{k}, \omega)=-e \int \mathrm{d}^{2} v v_{x} f(\boldsymbol{k}, \omega, \boldsymbol{v})$. We calculate

$$
\frac{4 \pi i}{\omega} J_{x}=\frac{4 \pi i}{\omega}(-e) \int v_{x} f \mathrm{~d}^{2} v=\frac{\omega_{p}^{2}}{\omega} \int \mathrm{d}^{2} v v_{x} \frac{E_{j} \frac{\partial f_{0}}{\partial v_{j}}}{\omega-\boldsymbol{k} \cdot \boldsymbol{v}}
$$

where we used Equation (8). The above quantity equals to $(\varepsilon-1) E_{x}$. Thus we have $D_{x}=\varepsilon E_{x}$. This statement can be most easily proved by assuming $f_{0}$ a Maxwellian. Use $\frac{\partial f_{0}}{\partial v_{j}}=-\frac{T}{m} v_{j} f_{0}$ and $\boldsymbol{E} \cdot \boldsymbol{v}=\frac{E_{x}}{k_{x}} \boldsymbol{k} \cdot \boldsymbol{v}$ to write for the last term

$$
\frac{4 \pi i}{\omega} J_{x}=-E_{x} \frac{\omega_{p}^{2}}{\omega} \frac{T}{m} \int \frac{v_{x}}{k_{x}} \frac{\boldsymbol{k} \cdot \boldsymbol{v}}{\omega-\boldsymbol{k} \cdot \boldsymbol{v}} f_{0} \mathrm{~d}^{2} v
$$

Put $\frac{\boldsymbol{k} \cdot \boldsymbol{v}}{\omega-\boldsymbol{k} \cdot \boldsymbol{v}}=-1+\frac{\omega}{\omega-\boldsymbol{k} \cdot \boldsymbol{v}}$. Then, (-1)-term vanishes upon integration, and we have

$$
\begin{aligned}
\frac{4 \pi i}{\omega} J_{x} & =-E_{x} \frac{\omega_{p}^{2}}{k_{x}} \frac{T}{m} \int \frac{v_{x} f_{0}}{\omega-\boldsymbol{k} \cdot \boldsymbol{v}} \mathrm{d}^{2} v=E_{x} \frac{\omega_{p}^{2}}{k_{x}} \int \frac{\frac{\partial f_{0}}{\partial v_{x}}}{\omega-\boldsymbol{k} \cdot \boldsymbol{v}} \mathrm{d}^{2} v \\
& =-E_{x} \omega_{p}^{2} \int \frac{f_{0} \mathrm{~d}^{2} v}{(\omega-\boldsymbol{k} \cdot \boldsymbol{v})^{2}}=E_{x} \frac{\omega_{p}^{2}}{k^{2}} \int \mathrm{d}^{2} v \frac{\boldsymbol{k} \cdot \frac{\partial f_{0}}{\partial \boldsymbol{v}}}{\omega-\boldsymbol{k} \cdot \boldsymbol{v}} \quad \text { q.e.d. }
\end{aligned}
$$

Using the above result, we obtain

$$
D_{x}^{(1)}(\boldsymbol{k}, \omega)=\varepsilon(\boldsymbol{k}, \omega) E_{x}^{(1)}(\boldsymbol{k}, \omega)=\frac{i k_{x}}{k^{2}}\left[N-4 \pi \rho_{0}(\boldsymbol{k}, \omega)\right]
$$

To invert Equation (15), we write

$$
D_{x}^{(1)}\left(x, k_{z}, \omega\right)=\int_{-\infty}^{\infty} \frac{\mathrm{d} k_{x}}{2 \pi} \mathrm{e}^{i k_{x} x} \frac{i k_{x}}{k^{2}}\left[N-\frac{8 \pi^{2}}{k_{0}^{2}} \rho_{1} \mathrm{e}^{-i k_{x} L_{1}} \delta\left(\omega+\omega_{1}\right) \mathrm{e}^{-i k_{z} S_{1}}+1 \rightarrow 2\right]
$$

In the above integral, we take the limit $x \rightarrow 0^{+}$. Evaluating the integral by residue theorem gives

$$
\lim _{x \rightarrow 0^{+}} \int_{-\infty}^{\infty} \mathrm{d} k_{x} \frac{k_{x} \mathrm{e}^{i k_{x} x}}{k_{x}^{2}+k_{z}^{2}}=i \pi
$$


Note that we set up the contour encircling the upper half plane since $x>0$. When $\operatorname{Re} k_{z}>0 \quad\left(\operatorname{Re} k_{z}<0\right)$, the relevant pole located in the upper $k_{x}$-plane is $k_{x}=i k_{z} \quad\left(k_{x}=-i k_{z}\right)$. In either case, the integral is found to be $i \pi$. Then, Equation (16) takes the form

$$
\begin{gathered}
D_{x}^{(1)}\left(0^{+}, k_{z}, \omega\right)=-\frac{N}{2}-\frac{4 \pi}{k_{0}^{2}} \mathrm{e}^{-i k_{2} S_{1}} i I\left(L_{1}, k_{z}\right) \rho_{1} \delta\left(\omega+\omega_{1}\right)+1 \rightarrow 2 \\
\text { where } I\left(L_{1}, k_{z}\right)=\int_{-\infty}^{\infty} \mathrm{d} \xi \frac{\xi \mathrm{e}^{-i \xi L_{1}}}{\xi^{2}+k_{z}^{2}}=-i \pi \mathrm{e}^{ \pm k_{z} L_{1}}
\end{gathered}
$$

where $+(-)$ sign corresponds to $\operatorname{Re} k_{z}<0 \quad\left(\operatorname{Re} k_{z}>0\right)$. The above equality can be easily proven by using the contour winding the lower half plane. Taking the limit $k_{z} \rightarrow 0$ gives the useful identity $\int_{-\infty}^{\infty} \mathrm{d} \xi \frac{\mathrm{e}^{-i \xi L_{1}}}{\xi}=-i \pi$, independently of $L_{1}(>0)$. Clearly, this integral manifests the nature of a step function. By equating the quantity on the right hand side of Equation (17) to the $x$-component of the vacuum electric field ( $\equiv E_{0}$ ), we obtain

$$
N=-2 E_{0}-\frac{8 \pi}{k_{0}^{2}} \rho_{1} \delta\left(\omega+\omega_{1}\right) \mathrm{e}^{-i k_{2} S_{1}} i I\left(L_{1}, k_{z}\right)+1 \rightarrow 2
$$

Using the above equation in Equation (13) gives

$$
\boldsymbol{E}^{(1)}(\boldsymbol{k}, \omega)=\frac{i \boldsymbol{k}}{k^{2} \varepsilon(\boldsymbol{k}, \omega)}\left[-2 E_{0}-\frac{8 \pi^{2}}{k_{0}^{2}} \rho_{1} \delta\left(\omega+\omega_{1}\right) \mathrm{e}^{-i k_{z} S_{1}}\left(\frac{i}{\pi} I\left(L_{1}, k_{z}\right)+\mathrm{e}^{-i k_{x} L_{1}}\right)+1 \rightarrow 2\right]
$$

For an infinite plasma without boundary, we have $N=0$ in Equation (13), and the plasma electric field is given by

$$
\boldsymbol{E}^{(1)}(\boldsymbol{k}, \omega)=\frac{i \boldsymbol{k}}{k^{2} \varepsilon(\boldsymbol{k}, \omega)}\left[-\frac{8 \pi^{2}}{k_{0}^{2}} \rho_{1} \delta\left(\omega+\omega_{1}\right) \mathrm{e}^{-i k_{z} S_{1}} \mathrm{e}^{-i k_{x} L_{1}}+1 \rightarrow 2\right]
$$

Note that in Equation (20), the $E_{0}$-term and $I\left(L_{1}, k_{z}\right)$-term are the boundary terms which are non-existent in an infinite plasma.

In the static situation where the electric field is nonpropagating, we put $k_{z}=0$ in Equation (20), and the electric field reduces to Equation (23) in Lee and Lee [5]:

$$
E^{(1)}(k, \omega)=\frac{-2 i}{k \varepsilon(k, \omega)}\left[E_{0}+\frac{4 \pi^{2}}{k_{0}^{2}} \rho_{1} \delta\left(\omega+\omega_{1}\right)\left(1+\mathrm{e}^{-i k L_{1}}\right)+1 \rightarrow 2\right]
$$

\section{Second Order Solution and Echo Occurrence}

Next, we deal with the second order equations, Equations (10) and (11). Using Equation (10) in Equation (11) yields, owing to the electrostatic nature of $\boldsymbol{E}^{(2)}$,

$$
\boldsymbol{E}^{(2)}(\omega, \boldsymbol{k})=\frac{4 \pi e^{2}}{m} \frac{\boldsymbol{k}}{k^{2} \varepsilon(\boldsymbol{k}, \omega)} \int \mathrm{d}^{2} v \frac{\boldsymbol{k} \cdot \boldsymbol{Q}}{(\omega-\boldsymbol{k} \cdot \boldsymbol{v})^{2}}
$$

where $\boldsymbol{Q}$ stands for

$$
\boldsymbol{Q}(\omega, \boldsymbol{k}, \boldsymbol{v})=\int_{-\infty}^{\infty} \frac{\mathrm{d} \omega^{\prime}}{2 \pi} \int_{-\infty}^{\infty} \frac{\mathrm{d} k_{x}^{\prime}}{2 \pi} \int_{-\infty}^{\infty} \frac{\mathrm{d} k_{z}^{\prime}}{2 \pi} \boldsymbol{E}^{(1)}\left(\omega-\omega^{\prime}, \boldsymbol{k}-\boldsymbol{k}^{\prime}\right) f^{(1)}\left(\omega^{\prime}, \boldsymbol{k}^{\prime}, \boldsymbol{v}\right)
$$

Substituting the first order solutions [Equations (8) and (20)], into the above equations, we can write $\boldsymbol{E}^{(2)}$ in the form,

$$
\boldsymbol{E}^{(2)}(\omega, \boldsymbol{k})=\frac{e^{3}}{2 \pi^{2} m^{2}} \frac{\boldsymbol{k}}{k^{2} \varepsilon(\boldsymbol{k}, \omega)} \int \frac{\mathrm{d}^{2} v}{(\omega-\boldsymbol{k} \cdot \boldsymbol{v})^{2}} \int \mathrm{d} k_{z}^{\prime} \int \mathrm{d} k_{x}^{\prime} \int \mathrm{d} \omega^{\prime} \times \frac{\boldsymbol{k} \cdot\left(\boldsymbol{k}-\boldsymbol{k}^{\prime}\right)}{\varepsilon\left(\omega-\omega^{\prime}, \boldsymbol{k}-\boldsymbol{k}^{\prime}\right)\left(\boldsymbol{k}-\boldsymbol{k}^{\prime}\right)^{2}} \frac{\boldsymbol{k}^{\prime} \cdot \frac{\mathrm{d} f_{0}}{\mathrm{~d} \boldsymbol{v}}}{k^{\prime 2} \varepsilon\left(\omega^{\prime}, \boldsymbol{k}^{\prime}\right)\left(\omega^{\prime}-\boldsymbol{k}^{\prime} \cdot \boldsymbol{v}\right)} A B,
$$




$$
\begin{gathered}
A=-2 E_{0}-\frac{8 \pi^{2}}{k_{0}^{2}} \rho_{1} \delta\left(\omega^{\prime}+\omega_{1}\right) \mathrm{e}^{-i k_{z} S_{1}}\left(\frac{i}{\pi} I\left(L_{1}, k_{z}^{\prime}\right)+\mathrm{e}^{-i k_{x}^{\prime} L_{1}}\right)+1 \rightarrow 2 \\
B=-2 E_{0}-\frac{8 \pi^{2}}{k_{0}^{2}} \rho_{1} \delta\left(\omega-\omega^{\prime}+\omega_{1}\right) \mathrm{e}^{-i\left(k_{z}-k_{z}^{\prime}\right) S_{1}}\left(\frac{i}{\pi} I\left(L_{1}, k_{z}-k_{z}^{\prime}\right)+\mathrm{e}^{-i\left(k_{x}-k_{x}^{\prime}\right) L_{1}}\right)+1 \rightarrow 2
\end{gathered}
$$

where $I$ stands for the exponential function as given by Equation (18). Since we don't know yet which sign should be chosen, we keep on using the symbol I. Equation (23) is to be used for investigation of echo occurrence. The various cross terms in the product $(\mathrm{AB})$ are the candidates of echo resonances to see if the condition for vanishing phase can be met.

We choose to investigate a cross term which is 1-term in A multiplied by 2-term in B. With this term, the $t$-inversion of Equation (23) can be easily carried out by simply putting $\omega^{\prime} \rightarrow-\omega_{1}$ and $\omega \rightarrow-\omega_{3}$ :

$$
\begin{aligned}
\boldsymbol{E}^{(2)}(t, \boldsymbol{k})= & \beta \mathrm{e}^{i \omega_{3} t} \mathrm{e}^{-i k_{z} S_{2}} \frac{\boldsymbol{k}}{k^{2} \varepsilon\left(-\omega_{3}, \boldsymbol{k}\right)} \int \frac{\mathrm{d}^{2} v}{\left(\omega_{3}+\boldsymbol{k} \cdot \boldsymbol{v}\right)^{2}} \int \mathrm{d} k_{z}^{\prime} \int \mathrm{d} k_{x}^{\prime} \\
& \times \frac{\boldsymbol{k} \cdot\left(\boldsymbol{k}-\boldsymbol{k}^{\prime}\right)}{\left(\boldsymbol{k}-\boldsymbol{k}^{\prime}\right)^{2} \varepsilon\left(-\omega_{2}, \boldsymbol{k}-\boldsymbol{k}^{\prime}\right)} \frac{\boldsymbol{k}^{\prime} \cdot \frac{\mathrm{d} f_{0}}{\mathrm{~d} \boldsymbol{v}}}{k^{\prime 2} \varepsilon\left(-\omega_{1}, \boldsymbol{k}^{\prime}\right)\left(\omega_{1}+\boldsymbol{k}^{\prime} \cdot \boldsymbol{v}\right)} \mathrm{e}^{i k_{z}^{\prime}\left(S_{2}-S_{1}\right)} \\
& \times\left(\frac{i}{\pi} I\left(L_{1}, k_{z}^{\prime}\right)+\mathrm{e}^{-i k_{x}^{\prime} L_{1}}\right)\left(\frac{i}{\pi} I\left(L_{2}, k_{z}-k_{z}^{\prime}\right)+\mathrm{e}^{-i\left(k_{x}-k_{x}^{\prime}\right) L_{2}}\right)
\end{aligned}
$$

where

$$
\omega_{3}=\omega_{1}+\omega_{2}, \beta=\frac{-16 \pi e^{3} \rho_{1} \rho_{2}}{k_{0}^{4} m^{2}}
$$

In the above equation, we can assume that the poles associated with the dielectric functions contribute negligibly in the $\int \mathrm{d} k$ - or $\int \mathrm{d} k^{\prime}$-integral. [The dominant contribution comes from the free-streaming poles.] Also we assume $f_{0}$ to be a Maxwellian. Then we have

$$
\frac{\boldsymbol{k}^{\prime} \cdot \frac{\mathrm{d} f_{0}}{\mathrm{~d} \boldsymbol{v}}}{\omega_{1}+\boldsymbol{k}^{\prime} \cdot \boldsymbol{v}}=-\frac{m}{T} f_{0}\left(1-\frac{\omega_{1}}{\omega_{1}+\boldsymbol{k}^{\prime} \cdot \boldsymbol{v}}\right)
$$

where 1 can be assumed to contribute nothing to the inversion integral in the following, due to phase mixing. Thus, Equation (26) can be further simplified as

$$
\begin{aligned}
\boldsymbol{E}^{(2)}(t, \boldsymbol{k})= & \frac{\beta m \omega_{1}}{T} \mathrm{e}^{i \omega_{3} t} \mathrm{e}^{-i k_{z} S_{2}} \frac{\boldsymbol{k}}{k^{2} \varepsilon\left(-\omega_{3}, \boldsymbol{k}\right)} \int \frac{\mathrm{d}^{2} v f_{0}}{\left(\omega_{3}+\boldsymbol{k} \cdot \boldsymbol{v}\right)^{2}} \int \mathrm{d} k_{z}^{\prime} \int \mathrm{d} k_{x}^{\prime} \\
& \times \frac{k \cdot\left(\boldsymbol{k}-\boldsymbol{k}^{\prime}\right)}{\left(\boldsymbol{k}-\boldsymbol{k}^{\prime}\right)^{2} \varepsilon\left(-\omega_{2}, \boldsymbol{k}-\boldsymbol{k}^{\prime}\right) k^{\prime 2} \varepsilon\left(-\omega_{1}, \boldsymbol{k}^{\prime}\right)\left(\omega_{1}+\boldsymbol{k}^{\prime} \cdot \boldsymbol{v}\right)} \\
& \times \mathrm{e}^{i k_{z}^{\prime}\left(S_{2}-S_{1}\right)}\left(\frac{i}{\pi} I\left(L_{1}, k_{z}^{\prime}\right)+\mathrm{e}^{-i k_{x}^{\prime} L_{1}}\right)\left(\frac{i}{\pi} I\left(L_{2}, k_{z}-k_{z}^{\prime}\right)+\mathrm{e}^{-i\left(k_{x}-k_{x}^{\prime}\right) L_{2}}\right)
\end{aligned}
$$

Let us write explicitly the inversion integral of Equation (28) with respect to $k$ :

$$
\begin{aligned}
\boldsymbol{E}^{(2)}(t, x, z)= & \frac{\beta m \omega_{1}}{4 \pi^{2} T} \mathrm{e}^{i \omega_{3} t} \int \frac{\mathrm{d}^{2} v f_{0}}{v_{x}^{2}} \int \mathrm{d} k_{z} \mathrm{e}^{i k_{z}\left(z-S_{2}\right)} \int \mathrm{d} k_{x} \frac{\boldsymbol{k}}{k^{2} \varepsilon\left(-\omega_{3}, \boldsymbol{k}\right)} \frac{\mathrm{e}^{i k_{x} x}}{\left(k_{x}+\frac{k_{z} v_{z}+\omega_{3}}{v_{x}}\right)^{2}} \\
& \times \int \mathrm{d} k_{z}^{\prime} \mathrm{e}^{i k_{z}^{\prime}\left(S_{2}-S_{1}\right)} \int \mathrm{d} k_{x}^{\prime} \frac{\boldsymbol{k} \cdot\left(\boldsymbol{k}-\boldsymbol{k}^{\prime}\right)}{\left(\boldsymbol{k}-\boldsymbol{k}^{\prime}\right)^{2} \varepsilon\left(-\omega_{2}, \boldsymbol{k}-\boldsymbol{k}^{\prime}\right) k^{\prime 2} \varepsilon\left(-\omega_{1}, \boldsymbol{k}^{\prime}\right)\left(\omega_{1}+\boldsymbol{k}^{\prime} \cdot \boldsymbol{v}\right)} \\
& \times\left(\frac{i}{\pi} I\left(L_{1}, k_{z}^{\prime}\right)+\mathrm{e}^{-i k_{x}^{\prime} L_{1}}\right)\left(\frac{i}{\pi} I\left(L_{2}, k_{z}-k_{z}^{\prime}\right)+\mathrm{e}^{-i\left(k_{x}-k_{x}^{\prime}\right) L_{2}}\right)
\end{aligned}
$$


This equation will be examined in view of the possibility of the vanishing phase.

(1) First, we shall consider the interference of two exponential terms in Equation (29): $\mathrm{e}^{-i k_{x}^{\prime} L_{1}} \mathrm{e}^{-i\left(k_{x}-k_{x}^{\prime}\right) L_{2}}=\mathrm{e}^{i k_{x}^{\prime}\left(L_{2}-L_{1}\right)} \mathrm{e}^{-i k_{x} L_{2}}$

The important singularities are: the double pole at $k_{x}=-\left(k_{z} v_{z}+\omega_{3}\right) / v_{x}$ and the simple poles associated respectively with $k^{2}=0$ and $k^{\prime 2}=0$ and $\omega_{1}+k_{x} v_{x}+k_{z} v_{z}=0$. We shall consider only these four poles. Singularities at $\varepsilon=0$ are not important. Therefore we can put

$$
\frac{\boldsymbol{k} \cdot\left(\boldsymbol{k}-\boldsymbol{k}^{\prime}\right)}{\left(\boldsymbol{k}-\boldsymbol{k}^{\prime}\right)^{2}}=\frac{1}{2}
$$

and all the $\varepsilon$ 's can be taken out of the integral. The residue at the double pole is obtained by taking $\partial / \partial k_{x}$ $\left[\right.$ Integrand $\left.\times\left(k_{x}+\frac{k_{z} v_{z}+\omega_{3}}{v_{x}}\right)^{2}\right]$ and substituting for $k_{x}=-\left(k_{z} v_{z}+\omega_{3}\right) / v_{x}$. Here it is sufficient to differentiate only the exponential functions because they yield asymptotically dominant result. [Or integrate by parts with respect to $k_{x}$.] Thus let us calculate

$$
\begin{aligned}
\Phi= & \frac{i\left(x-L_{2}\right)}{v_{x}} \int \mathrm{d} k_{z} \mathrm{e}^{i k_{z}\left(z-S_{2}\right)} \int \mathrm{d} k_{x} \frac{\boldsymbol{k}}{k^{2}} \frac{\mathrm{e}^{i k_{x}\left(x-L_{2}\right)}}{k_{x}+\left(k_{z} v_{z}+\omega_{3}\right) / v_{x}} \\
& \times \int \mathrm{d} k_{z}^{\prime} \frac{\mathrm{e}^{i k_{z}^{\prime}\left(S_{2}-S_{1}\right)}}{k^{\prime 2}} \int \mathrm{d} k_{x}^{\prime} \frac{\mathrm{e}^{i k_{x}^{\prime}\left(L_{2}-L_{1}\right)}}{k_{x}^{\prime}+\left(k_{z}^{\prime} v_{z}+\omega_{1}\right) / v_{x}}
\end{aligned}
$$

Then $\boldsymbol{E}^{(2)}$ is obtained by $\mathrm{e}^{\mathrm{i} \omega_{3} t} \int \mathrm{d}^{2} v \Phi f_{0} / v_{x}^{2}$, suppressing the unessential factor.

Integral $\int \mathrm{d} k_{x}^{\prime}$ can be easily done by picking up the pole at

$$
k_{x}^{\prime}=-\frac{k_{z}^{\prime} v_{z}+\omega_{1}}{v_{x}}
$$

For definiteness we assume $L_{2}>L_{1}$. Then the contour in $k_{x}^{\prime}$-plane should encircle the upper half $k_{x}^{\prime}$-plane, and in order for the pole to lie in the upper $k_{x}^{\prime}$-plane, the imaginary part of $\left(k_{z}^{\prime} v_{z} / v_{x}\right)$ should be negative. Now $k^{\prime 2}$ is only a function of $k_{z}^{\prime}$ per Equation (31), and we can write the second part of Equation (30) as

$$
\begin{aligned}
J^{\prime} & \equiv \int \mathrm{d} k_{z}^{\prime} \frac{\mathrm{e}^{i k_{z}^{\prime}\left(S_{2}-S_{1}\right)}}{k^{\prime 2}} \int \mathrm{d} k_{x}^{\prime} \frac{\mathrm{e}^{i k_{x}^{\prime}\left(L_{2}-L_{1}\right)}}{k_{x}^{\prime}+\left(k_{z}^{\prime} v_{z}+\omega_{1}\right) / v_{x}} \\
& =-\frac{\pi v_{x}}{\omega_{1}} \mathrm{e}^{-i\left(L_{2}-L_{1}\right) \omega_{1} / v_{x}} \int \mathrm{d} k_{z}^{\prime} \mathrm{e}^{i \theta^{\prime}}\left(\frac{1}{k_{z}^{\prime}-k_{z+}^{\prime}}-\frac{1}{k_{z}^{\prime}-k_{z-}^{\prime}}\right) H\left(-\frac{v_{z}}{v_{x}} \operatorname{Im} k_{z}^{\prime}\right)
\end{aligned}
$$

where

$$
\begin{gathered}
k_{z \pm}^{\prime}=-\frac{\omega_{1}}{v^{2}}\left(v_{z} \pm i v_{x}\right) \\
\theta^{\prime}=S_{2}-S_{1}+\left(L_{1}-L_{2}\right) \frac{v_{z}}{v_{x}}
\end{gathered}
$$

and $H(x)$ is a step function; $H(x)=1$ for $x>0$ and $H(x)=0$ for $x<0$.

The contour in $\int \mathrm{d} k_{z}^{\prime}$-integral depends on the sign of $\theta^{\prime}$ : when $\theta^{\prime}>0 \quad\left(\theta^{\prime}<0\right)$, the contour must wind the upper (lower) $k_{z}^{\prime}$-plane. The location of the poles depends upon the sign of $v_{x}$. Sorting out the relevant cases, we carry out the integral for $J^{\prime}$ :

$$
\begin{aligned}
& \theta^{\prime}>0: J^{\prime}=\frac{-2 i \pi^{2} v_{x}}{\omega_{1}} \mathrm{e}^{i\left(L_{1}-L_{2}\right) \omega_{1} / v_{x}}\left[\mathrm{e}^{i \theta^{\prime} k_{z+}^{\prime}} H\left(v_{z}\right) H\left(-v_{x}\right)-\mathrm{e}^{i \theta^{\prime} k_{z}^{\prime}} H\left(-v_{z}\right) H\left(v_{x}\right)\right] \\
& \theta^{\prime}<0: J^{\prime}=\frac{2 i \pi^{2} v_{x}}{\omega_{1}} \mathrm{e}^{i\left(L_{1}-L_{2}\right) \omega_{1} / v_{x}}\left[\mathrm{e}^{i \theta^{\prime} k_{z+}^{\prime}} H\left(v_{z}\right) H\left(v_{x}\right)-\mathrm{e}^{i \theta^{\prime} k_{z-}^{\prime}} H\left(-v_{z}\right) H\left(-v_{x}\right)\right]
\end{aligned}
$$


Next, taking on the first part of the integral in Equation (30) $\left(\int \mathrm{d} k_{z}(\cdots)\right)$, we have two cases:

1) $x<L_{2}$

In this case, the $k_{x}$ contour must encircle the lower half plane and the $k_{x}$-integral does not vanish under the provision $\operatorname{Im}\left(k_{z} v_{z} / v_{x}\right)>0$. Then, the integral can be written as

$$
\begin{aligned}
\boldsymbol{J} & \equiv \int \mathrm{d} k_{\mathrm{z}} \mathrm{e}^{i k_{z}\left(z-S_{2}\right)} \int \mathrm{d} k_{x} \frac{\boldsymbol{k}}{k^{2}} \frac{\mathrm{e}^{i k_{x}\left(x-L_{2}\right)}}{k_{x}+\left(k_{z} v_{z}+\omega_{3}\right) / v_{x}} \\
& =\frac{\pi v_{x}}{\omega_{1}} \mathrm{e}^{-i\left(x-L_{2}\right) \omega_{3} / v_{x}} \int \mathrm{d} k_{\mathrm{z}} \mathrm{e}^{\mathrm{i} \theta}\left(\frac{1}{k_{z}-k_{z+}}-\frac{1}{k_{z}-k_{z-}}\right) H\left(\frac{v_{z}}{v_{x}} \operatorname{Im} k_{z}\right)\left[-\hat{\boldsymbol{x}} \frac{k_{z} v_{z}+\omega_{3}}{v_{x}}+\boldsymbol{z} \hat{k}_{z}\right]
\end{aligned}
$$

where

$$
\begin{aligned}
& k_{z \pm}=-\frac{\omega_{3}}{v^{2}}\left(v_{z} \pm i v_{x}\right) \\
& \theta=z-S_{2}+\left(L_{2}-x\right) \frac{v_{z}}{v_{x}}
\end{aligned}
$$

Analogously to the foregoing calculation in $J^{\prime}$, the above integral depends on the sign of $\theta$ :

$$
\begin{gathered}
\theta>0: \boldsymbol{J}=\frac{2 i \pi^{2} v_{x}}{\omega_{3}} \mathrm{e}^{i\left(L_{2}-x\right) \omega_{3} / v_{x}}\left[\boldsymbol{k}_{z+} \mathrm{e}^{i \theta k_{z+}} H\left(-v_{x}\right) H\left(-v_{z}\right)-\boldsymbol{k}_{z-} \mathrm{e}^{i \theta k_{z-}} H\left(v_{x}\right) H\left(v_{z}\right)\right] \\
\theta<0: \boldsymbol{J}=\frac{-2 i \pi^{2} v_{x}}{\omega_{3}} \mathrm{e}^{i\left(L_{2}-x\right) \omega_{3} / v_{x}}\left[\boldsymbol{k}_{z+} \mathrm{e}^{i \theta k_{z+}} H\left(v_{x}\right) H\left(-v_{z}\right)-\boldsymbol{k}_{z-} \mathrm{e}^{i \theta k_{z-}} H\left(-v_{x}\right) H\left(v_{z}\right)\right]
\end{gathered}
$$

2) $x>L_{2}$

Repeating a similar analysis, we obtain

$$
\begin{aligned}
& \theta>0: \boldsymbol{J}=\frac{-2 i \pi^{2} v_{x}}{\omega_{3}} \mathrm{e}^{i\left(L_{2}-x\right) \omega_{3} / v_{x}}\left[\boldsymbol{k}_{z+} \mathrm{e}^{i \theta k_{z+}} H\left(-v_{x}\right) H\left(v_{z}\right)-\boldsymbol{k}_{z-} \mathrm{e}^{i \theta k_{z-}} H\left(v_{x}\right) H\left(-v_{z}\right)\right] \\
& \theta<0: \boldsymbol{J}=\frac{2 i \pi^{2} v_{x}}{\omega_{3}} \mathrm{e}^{i\left(L_{2}-x\right) \omega_{3} / v_{x}}\left[\boldsymbol{k}_{z+} \mathrm{e}^{i \theta k_{z+}} H\left(v_{x}\right) H\left(v_{z}\right)-\boldsymbol{k}_{z-} \mathrm{e}^{\mathrm{i} \theta k_{z-}} H\left(-v_{x}\right) H\left(-v_{z}\right)\right]
\end{aligned}
$$

where

$$
\boldsymbol{k}_{z \pm}=k_{z \pm}(\mp i \hat{\boldsymbol{x}}+\hat{\mathbf{z}})
$$

Now, we have to multiply $\boldsymbol{J}$ and $J^{\prime}$. In doing it, note that $H(x) H(x)=H(x)$ and $H(x) H(-x)=0$. Nonzero results surviving the velocity integral are obtained in the following four cases:

a) $x<L_{2}, \theta<0, \theta^{\prime}>0$; b) $x<L_{2}, \theta>0, \theta^{\prime}<0$; c) $x>L_{2}, \theta>0, \theta^{\prime}>0$; d) $x>L_{2}, \theta<0$, $\theta^{\prime}<0$

Let us first consider case a). Using Equations (35) and (41), we obtain

$$
\begin{aligned}
\Phi= & \frac{\left(2 \pi^{2} v_{x}\right)^{2}}{\omega_{1} \omega_{3}} \frac{i\left(x-L_{2}\right)}{v_{x}}\left[H\left(v_{x}\right) H\left(-v_{z}\right) k_{z+} \exp \left(i \theta_{0}+i \theta k_{z+}+i \theta^{\prime} k_{z-}^{\prime}\right)\right. \\
& \left.+H\left(-v_{x}\right) H\left(v_{z}\right) k_{z-} \exp \left(i \theta_{0}+i \theta k_{z-}+i \theta^{\prime} k_{z+}^{\prime}\right)\right]
\end{aligned}
$$

where

$$
\theta_{0}=\left(L_{2}-x\right) \frac{\omega_{3}}{v_{x}}+\left(L_{1}-L_{2}\right) \frac{\omega_{1}}{v_{x}}
$$

Using Equations (34), (39), and (46), we can obtain the exponential phases:

$$
i \theta_{0}+i \theta k_{z-}+i \theta^{\prime} k_{z+}^{\prime}=\varphi_{r}^{\prime}+i \varphi_{i}
$$




$$
i \theta_{0}+i \theta k_{z+}+i \theta^{\prime} k_{z-}^{\prime}=-\varphi_{r}^{\prime}+i \varphi_{i}
$$

with

$$
\begin{aligned}
& \varphi_{r}^{\prime}=\frac{1}{v^{2}}\left[v_{x} \omega_{3}\left(S_{2}-z\right)+v_{x} \omega_{1}\left(S_{2}-S_{1}\right)+v_{z} \omega_{3}\left(x-L_{2}\right)+v_{z} \omega_{1}\left(L_{1}-L_{2}\right)\right] \\
& \varphi_{i}=\frac{1}{v^{2}}\left[v_{z} \omega_{3}\left(S_{2}-z\right)+v_{z} \omega_{1}\left(S_{1}-S_{2}\right)+v_{x} \omega_{3}\left(L_{2}-x\right)+v_{x} \omega_{1}\left(L_{1}-L_{2}\right)\right]
\end{aligned}
$$

Thus Equation (45) can be written in the form

$$
\Phi=\frac{\left(2 \pi^{2} v_{x}\right)^{2}}{\omega_{1} \omega_{3}} \frac{i\left(x-L_{2}\right)}{v_{x}} \mathrm{e}^{i \varphi_{i}}\left[H\left(v_{x}\right) H\left(-v_{z}\right) \boldsymbol{k}_{z+} \exp \left(-\varphi_{r}^{\prime}\right)+H\left(-v_{x}\right) H\left(v_{z}\right) \boldsymbol{k}_{z-} \exp \left(\varphi_{r^{\prime}}\right)\right]
$$

Therefore the velocity integrals in Equation (29) survive the phase mixing when $\varphi_{i}=0$, that is,

$$
\begin{gathered}
\omega_{3}\left(z-S_{2}\right)+\omega_{1}\left(S_{2}-S_{1}\right)=0, \omega_{3}\left(L_{2}-x\right)+\omega_{1}\left(L_{1}-L_{2}\right)=0 \\
\text { or } x=\frac{\omega_{1} L_{1}+\omega_{2} L_{2}}{\omega_{1}+\omega_{2}}, z=\frac{\omega_{1} S_{1}+\omega_{2} S_{2}}{\omega_{1}+\omega_{2}}
\end{gathered}
$$

where an echo is given rise to. The electric field $\boldsymbol{E}^{(2)}$ can be obtained by velocity integral in the form (see Equation (29))

$$
\boldsymbol{E}^{(2)}(t, x, z)=\left[\int_{0}^{\infty} \mathrm{d} v_{x} \int_{-\infty}^{0} \mathrm{~d} v_{z} \boldsymbol{k}_{z+} \mathrm{e}^{-\varphi_{r}^{\prime}}+\int_{-\infty}^{0} \mathrm{~d} v_{x} \int_{0}^{\infty} \mathrm{d} v_{z} k_{z-} \mathrm{e}^{\varphi_{r}^{\prime}}\right](\cdots)
$$

where $(\cdots)$ denotes the obvious integrand.

Next, let us calculate case (b). Using Equations (36) and (40) gives

$$
\begin{aligned}
\Phi= & \frac{\left(2 \pi^{2} v_{x}\right)^{2}}{\omega_{1} \omega_{3}} \frac{i\left(x-L_{2}\right)}{v_{x}}\left[H\left(-v_{x}\right) H\left(-v_{z}\right) \boldsymbol{k}_{z+} \exp \left(i \theta_{0}+i \theta k_{z+}+i \theta^{\prime} k_{z-}^{\prime}\right)\right. \\
& \left.+H\left(v_{x}\right) H\left(v_{z}\right) \boldsymbol{k}_{z-} \exp \left(i \theta_{0}+i \theta k_{z-}+i \theta^{\prime} k_{z+}^{\prime}\right)\right]
\end{aligned}
$$

This equation is identical with Equation (45) if $H\left(v_{x}\right)$ and $H\left(-v_{x}\right)$ are interchanged in the latter. Thus, this case can give rise to an echo at the same spot as predicted by Equation (53). The corresponding electric field is obtained by a similar velocity integral to Equation (54) but over different range of $v_{x}$.

The cases (a) and (b) predict the same echo spot because they yield the same imaginary phase $\varphi_{i}$. One more task: the various inequality conditions set forth to specify the contour in the contour integrations need to be checked against the echo coordinate found in Equation (54). Let us consider the inequalities $\theta<0$ and $\theta^{\prime}>0$ postulated in the case (a). Using Equation (52), the inequality $\theta<0$ can be written in the form

$$
S_{1}-S_{2}+\frac{v_{z}}{v_{x}}\left(L_{2}-L_{1}\right)<0
$$

which is the condition $\theta^{\prime}>0$. Therefore the conditions $\theta<0$ and $\theta^{\prime}>0$ imply each other. Also we can ascertain that the echo $x$-coordinate is in accord with the condition $x<L_{2}$. So in cases (a) and (b), the premise and the result are consistent. For the cases of (c) and (d), we state without repeating a similar algebra that the imaginary part of the phase is still obtained by Equation (50) [the real part of the phase is different]. Although the echo spot is predicted by the same equation as Equation (53), these cases of (c) and (d) are not acceptable because the conditions $\theta>0$ and $\theta^{\prime}>0$ or $\theta<0$ and $\theta^{\prime}<0$ are contradictory to each other. We have the conclusion: an echo occurs where $x<L_{2}$ and the echo coordinates are predicted by Equation (53).

(2) Next, we consider the product of two boundary terms, $I\left(L_{1}, k_{z}^{\prime}\right) I\left(L_{2}, k_{z}-k_{z}^{\prime}\right)$ in Equation (29):

$$
\begin{aligned}
\boldsymbol{E}^{(2)}(t, x, z)= & C \mathrm{e}^{\mathrm{i} \omega_{3} t} \int \frac{\mathrm{d}^{2} v f_{0}}{v_{x}^{2}} \int \mathrm{d} k_{z} \exp \left[i k_{z}\left(z-S_{2}\right)\right] \int \mathrm{d} k_{x} \frac{\boldsymbol{k}}{k^{2}} \frac{\exp \left[i k_{x}\right]}{\left(k_{x}+\left(k_{z} v_{z}+\omega_{3}\right) / v_{x}\right)^{2}} \\
& \times \int \mathrm{d} k_{z}^{\prime} \mathrm{e}^{i k_{z}^{\prime}\left(S_{2}-S_{1}\right)} I\left(L_{1}, k_{z}^{\prime}\right) I\left(L_{2}, k_{z}-k_{z}^{\prime}\right) \int \frac{\mathrm{d} k_{x}^{\prime}}{k^{\prime 2}\left(\omega_{1}+\boldsymbol{k}^{\prime} \cdot \boldsymbol{v}\right)}
\end{aligned}
$$


where $C$ is a nonessential constant factor. For definiteness, we assume $S_{2}-S_{1}>0 . \int \mathrm{d} k_{x}^{\prime}$-integral and $\int \mathrm{d} k_{x}$ integral can be done easily by picking up the relevant poles, and we can write

$$
\begin{aligned}
\boldsymbol{E}^{(2)}(t, x, z)= & i x C \mathrm{e}^{\mathrm{i} \omega_{3} t} \int \frac{\mathrm{d}^{2} v f_{0}}{v_{x}^{3}} \exp \left[-i x \frac{\omega_{3}}{v_{x}}\right] \int \mathrm{d} k_{z} \frac{\boldsymbol{k}}{k^{2}} \exp \left[i k_{z}\left(z-S_{2}-x \frac{v_{x}}{v_{z}}\right)\right] \\
& \times \int \frac{\mathrm{d} k_{z}^{\prime}}{k^{\prime 2}} \mathrm{e}^{i k_{z}^{\prime}\left(S_{2}-S_{1}\right)} I\left(L_{1}, k_{z}^{\prime}\right) I\left(L_{2}, k_{z}-k_{z}^{\prime}\right)
\end{aligned}
$$

where

$$
\begin{aligned}
& k^{\prime 2}=k_{z}^{\prime 2}+\frac{\left(\omega_{1}+k_{z}^{\prime} v_{z}\right)^{2}}{v_{x}} \\
& k=-x \frac{k_{z} v_{z}+\omega_{3}}{v_{x}}+z k_{z}
\end{aligned}
$$

The contour of $\int \mathrm{d} k_{z}^{\prime}$-integral should encircle the upper $k_{z}^{\prime}$-plane. Since the relevant singularity should be located in the upper $k_{z}^{\prime}$-plane, the residue is calculated from $k_{z}^{\prime}=k_{z+}^{\prime}$ for $v_{x}<0$ and $k_{z}^{\prime}=k_{z-}^{\prime}$ for $v_{x}>0$. $k_{z \pm}^{\prime}$ are defined in Equation (33). Then the last integral ( $\left.\int d k_{z}^{\prime}\right)$ in Equation (57) can be carried out in the form

$$
\begin{aligned}
\int \mathrm{d} k_{z}^{\prime}(\cdots)= & \frac{-\pi v_{x}}{\omega_{1}}\left[\exp \left[i k_{z+}^{\prime}\left(S_{2}-S_{1}\right)\right] I\left(L_{1}, k_{z+}^{\prime}\right) I\left(L_{2}, k_{z}-k_{z+}^{\prime}\right) H\left(-v_{x}\right)\right. \\
& \left.-\exp \left[i k_{z-}^{\prime}\left(S_{2}-S_{1}\right)\right] I\left(L_{1}, k_{z-}^{\prime}\right) I\left(L_{2}, k_{z}-k_{z-}^{\prime}\right) H\left(v_{x}\right)\right] \equiv J\left(k_{z}\right)
\end{aligned}
$$

where

$$
I\left(L_{1}, k_{z \pm}^{\prime}\right)=-i \pi\left(\exp \left[L_{1} k_{z \pm}^{\prime}\right] H\left(v_{z}\right)+\exp \left[-L_{1} k_{z \pm}^{\prime}\right] H\left(-v_{z}\right)\right) \text { (see Equation (18)) }
$$

To carry out $\int \mathrm{d} k_{z}(\cdots)$ in Equation (59), let us assume that $z-S_{2}-x \frac{v_{z}}{v_{x}} \equiv \vartheta<0$.

Now we are ready to evaluate $\int \mathrm{d} k_{z}$-integral in Equation (59):

$$
\begin{aligned}
\int \mathrm{d} k_{z}(\cdots) & =\int \mathrm{d} k_{z} \frac{\boldsymbol{k} \mathrm{e}^{i k_{z} \vartheta}}{k^{2}} J\left(k_{z}\right)=\frac{i v_{x}}{2 \omega_{3}} \int \mathrm{d} k_{z} \boldsymbol{k} J\left(k_{z}\right)\left[\frac{\mathrm{e}^{i k_{z} \vartheta}}{k_{z}-k_{z+}}-\frac{\mathrm{e}^{i k_{z} \vartheta}}{k_{z}-k_{z-}}\right] \\
& =\frac{\pi v_{x}}{\omega_{3}}\left[\boldsymbol{k}\left(k_{z+}\right) \mathrm{e}^{i k_{z+} \vartheta} J\left(k_{z+}\right) H\left(v_{x}\right)-\boldsymbol{k}\left(k_{z-}\right) \mathrm{e}^{i k_{z-} \vartheta} J\left(k_{z-}\right) H\left(-v_{x}\right)\right]
\end{aligned}
$$

Using Equation (60) in Equation (62) yields

$$
\begin{aligned}
\int \mathrm{d} k_{z}(\cdots)= & \frac{\pi^{2} v_{x}^{2}}{\omega_{1} \omega_{3}}\left[\boldsymbol{k}\left(k_{z+}\right) \exp \left[i k_{z+} \vartheta\right] \exp \left[i k_{z-}^{\prime}\left(S_{2}-S_{1}\right)\right] I\left(L_{1}, k_{z-}^{\prime}\right) I\left(L_{2}, k_{z+}-k_{z-}^{\prime}\right) H\left(v_{x}\right)\right. \\
& \left.+\boldsymbol{k}\left(k_{z-}\right) \exp \left[i k_{z-} \vartheta\right] \exp \left[i k_{z+}^{\prime}\left(S_{2}-S_{1}\right)\right] I\left(L_{1}, k_{z+}^{\prime}\right) I\left(L_{2}, k_{z-}-k_{z+}^{\prime}\right) H\left(-v_{x}\right)\right]
\end{aligned}
$$

where we have $\operatorname{Re}\left(k_{z+}-k_{z-}^{\prime}\right)=\operatorname{Re}\left(k_{z-}-k_{z+}^{\prime}\right)=-\frac{\omega_{2}}{v^{2}} v_{z}$. Therefore, we obtain

$$
\begin{aligned}
& I\left(L_{2}, k_{z+}-k_{z-}^{\prime}\right)=-i \pi\left(\exp \left[L_{2}\left(k_{z+}-k_{z-}^{\prime}\right)\right] H\left(v_{z}\right)+\exp \left[-L_{2}\left(k_{z+}-k_{z-}^{\prime}\right)\right] H\left(-v_{z}\right)\right) \\
& I\left(L_{2}, k_{z-}-k_{z+}^{\prime}\right)=-i \pi\left(\exp \left[L_{2}\left(k_{z-}-k_{z+}^{\prime}\right)\right] H\left(v_{z}\right)+\exp \left[-L_{2}\left(k_{z-}-k_{z+}^{\prime}\right)\right] H\left(-v_{z}\right)\right)
\end{aligned}
$$

The above two equations and Equation (61) yield

$$
I\left(L_{1}, k_{z-}^{\prime}\right) I\left(L_{2}, k_{z+}-k_{z-}^{\prime}\right)=-\pi^{2}\left[\exp \left[L_{2} k_{z+}+\left(L_{1}-L_{2}\right) k_{z-}^{\prime}\right] H\left(v_{z}\right)+\exp \left[-L_{2} k_{z+}-\left(L_{1}-L_{2}\right) k_{z-}^{\prime}\right] H\left(-v_{z}\right)\right]
$$




$$
I\left(L_{1}, k_{z+}^{\prime}\right) I\left(L_{2}, k_{z-}-k_{z+}^{\prime}\right)=-\pi^{2}\left[\exp \left[L_{2} k_{z-}+\left(L_{1}-L_{2}\right) k_{z+}^{\prime}\right] H\left(v_{z}\right)+\exp \left[-L_{2} k_{z-}-\left(L_{1}-L_{2}\right) k_{z+}^{\prime}\right] H\left(-v_{z}\right)\right]
$$

Now we are ready to carry out the velocity integral in Equation (59) by substituting Equation (63) into it. Because of the step functions $H\left(v_{x}\right)$ and $H\left(v_{z}\right)$, the velocity integral consists of four parts corresponding to $H\left( \pm v_{x}\right) H\left( \pm v_{z}\right)$. Since we are interested in the echo spots, we pay attention only to the exponential phases:

$$
H\left(v_{x}\right) H\left(-v_{z}\right) \mathrm{e}^{\varphi_{1}}, H\left(v_{x}\right) H\left(v_{z}\right) \mathrm{e}^{\varphi_{2}}, H\left(-v_{x}\right) H\left(v_{z}\right) \mathrm{e}^{\varphi_{3}}, H\left(-v_{x}\right) H\left(-v_{z}\right) \mathrm{e}^{\varphi_{4}}
$$

Straightly we can identify:

$$
\begin{aligned}
& \varphi_{1}=-i x \frac{\omega_{3}}{v_{x}}+i k_{z+} \vartheta+i k_{z-}^{\prime}\left(S_{2}-S_{1}\right)-L_{2} k_{z+}+\left(L_{2}-L_{1}\right) k_{z-}^{\prime} \\
& \varphi_{2}=-i x \frac{\omega_{3}}{v_{x}}+i k_{z+} \vartheta+i k_{z-}^{\prime}\left(S_{2}-S_{1}\right)+L_{2} k_{z+}+\left(L_{1}-L_{2}\right) k_{z-}^{\prime} \\
& \varphi_{3}=-i x \frac{\omega_{3}}{v_{x}}+i k_{z-} \vartheta+i k_{z+}^{\prime}\left(S_{2}-S_{1}\right)+L_{2} k_{z-}+\left(L_{1}-L_{2}\right) k_{z+}^{\prime} \\
& \varphi_{4}=-i x \frac{\omega_{3}}{v_{x}}+i k_{z-} \vartheta+i k_{z+}^{\prime}\left(S_{2}-S_{1}\right)-L_{2} k_{z-}+\left(L_{2}-L_{1}\right) k_{z+}^{\prime}
\end{aligned}
$$

From above, the imaginary phases are obtained as

$$
\operatorname{Im} \varphi_{1}=\operatorname{Im} \varphi_{3}=\frac{v_{z}}{v^{2}}\left[\omega_{1}\left(S_{1}-S_{2}\right)-\omega_{3}\left(z-S_{2}\right)\right]+\frac{v_{x}}{v^{2}}\left[L_{2}\left(\omega_{1}+\omega_{3}\right)-L_{1} \omega_{1}-x \omega_{3}\right]
$$

$\operatorname{Im} \varphi_{2}=\operatorname{Im} \varphi_{4}$ is obtained from $\operatorname{Im} \varphi_{1}$ by replacing $L_{1} \rightarrow-L_{1}$ and $L_{2} \rightarrow-L_{2}$. Putting $\operatorname{Im} \varphi_{1,2}=0$, we obtain the echo spots as

$$
\begin{gathered}
z_{\text {echo }}=\frac{\omega_{1} S_{1}+\omega_{2} S_{2}}{\omega_{3}} \\
x_{\text {echo }}=\frac{ \pm L_{2}\left(2 \omega_{1}+\omega_{2}\right) \mp L_{1} \omega_{1}}{\omega_{3}}
\end{gathered}
$$

In Equation (75), $x_{\text {echo }}$ corresponding to upper signs and $x_{\text {echo }}$ corresponding to lower signs are mutually exclusive because if one of them is inside the plasma the other is necessarily is outside the plasma. We add that the condition $\vartheta<0$ amounts to $\frac{\omega_{1}}{\omega_{3}}\left(S_{1}-S_{2}\right)<x \frac{v_{z}}{v_{x}}$, which poses no problem in as much as we have ample liberty in choosing the sign of $v_{z} / v_{x}$.

\section{Discussion}

In Section 3, the plasma electric field was determined in terms of the vacuum electric field. Judicious application of the boundary conditions at the interface enables one to determine the plasma electric field entirely in terms of the external charges without introducing the vacuum electric field $E_{0}$. Inverting Equation (13), we can write

$$
\begin{gathered}
D_{x}(0, \omega)=-\frac{N}{2}-i \frac{4 \pi}{k_{0}^{2}} \rho_{1} \delta\left(\omega+\omega_{1}\right) \mathrm{e}^{-i k_{z} S_{1}} I\left(k_{z}, L_{1}\right) \\
E_{z}(0, \omega)=i N \int \frac{k_{z}}{k^{2} \varepsilon} \frac{\mathrm{d} k_{x}}{2 \pi}-i \frac{4 \pi}{k_{0}^{2}} \rho_{1} \delta\left(\omega+\omega_{1}\right) \mathrm{e}^{-i k_{z} S_{1}} I_{\varepsilon}\left(k_{z}, L_{1}\right) \\
\text { where } I_{\varepsilon}\left(k_{z}, L_{1}\right)=\int \frac{k_{z} \mathrm{~d} k_{x}}{k^{2} \varepsilon} \mathrm{e}^{-i k_{x} L_{1}}
\end{gathered}
$$

Next, we turn to the vacuum solution.

$$
E_{x}\left(x, k_{z}\right)=-B^{\prime} k_{z} \mathrm{e}^{k_{z} x} \equiv E_{0} \mathrm{e}^{k_{z} x}
$$


where $E_{0}$ is the vacuum electric field, the quantity designated by the same symbol $E_{0}$ in Equation (19).

$$
E_{z}\left(x, k_{z}\right)=-i B^{\prime} k_{z} e^{k_{z} x}
$$

Continuity of $E_{z}$ across the interface $x=0$ gives that Equation (22) equals to $-i B^{\prime} k_{z}$. Also continuity of $D_{x}$ across $x=0$ yields that Equation (20) equals to $-B^{\prime} k_{z}$. Eliminating $B^{\prime}$ between these two equations gives $\mathrm{N}$ in the form

$$
N=\frac{\frac{8 \pi}{k_{0}^{2}} \rho_{1} \delta\left(\omega+\omega_{1}\right) \mathrm{e}^{-i k_{z} S_{1}}\left(I_{\varepsilon}\left(k_{z}, L_{1}\right)-i I\left(k_{z}, L_{1}\right)\right)+1 \rightarrow 2}{1+\int \frac{\mathrm{d} k_{x}}{\pi} \frac{k_{z}}{k^{2} \varepsilon}}
$$

Substituting the above equation into Equation (13) yields

$$
\boldsymbol{E}(\boldsymbol{k}, \omega)=\frac{8 \pi^{2}}{k_{0}^{2}} \frac{i \boldsymbol{k}}{k^{2} \varepsilon} \rho_{1} \delta\left(\omega+\omega_{1}\right) \mathrm{e}^{-i k_{z} S_{1}} \frac{-i I\left(k_{z}, L_{1}\right) / \pi-\mathrm{e}^{-i k_{x} L_{1}}}{1+\int \frac{\mathrm{d} k_{x}}{\pi} \frac{k_{z}}{k^{2} \varepsilon}}
$$

Equation (82) should be compared with Equation (20). Eliminating the vacuum field introduces the denominator $1+\int \frac{d k_{x}}{\pi} \frac{k_{z}}{k^{2} \varepsilon}$ in Equation (82). In fact, the relation

$$
1+\int \frac{\mathrm{d} k_{x}}{\pi} \frac{k_{z}}{k^{2} \varepsilon}=0
$$

is the electrostatic dispersion relation of the surface wave in a semi-bounded plasma [10].

In the investigation of echo occurrence, $E_{0}$ in Equation (20) can be discarded because echoes are given rise to by interference of influences of the external charges. This amounts to saying that the denominator $\left(1+\int \frac{\mathrm{d} k_{x}}{\pi} \frac{k_{z}}{k^{2} \varepsilon}\right)$ doesn't play any role in the determination of echo locations.

Equations (53) and (74) and (75) are the main results of this work in locating the echo spots associated with the surface wave in a semi-bounded plasma launched by the oscillating external charges at $(x, z)=\left(L_{1}, S_{1}\right)$ and $\left(L_{2}, S_{2}\right)$. In the static situation, the $z$-coordinate is irrelevant. The echo spot given by Equation (53) corresponds to $x_{\text {echo }}$ in Equation (45) in Lee and Lee [5]. The echo spot given by Equations (74) and (75) is surface wave-proper. Our search for the echo spots are not exhaustive; we put aside many other product terms in (AB) in Equations (24) and (25). It appears that we have diversity of echoes in a bounded plasma, which was also experimentally reported [7]. The diversity seems to be due to reflections of the wave at the interface.

In reality, bounded plasmas are usual rather than exceptional. Important literatures to get acquainted with this field are References [8] and [9], among others. Surface wave dispersion relation in a plasma slab is derived in Ref. [10]. An exact nonlinear solution of a surface wave excited by external charges is obtained in Ref. [11].

\section{Acknowledgements}

Hee J. Lee thanks Professor L. Stenflo for correspondence. The work of MJL is supported by the National R\&D Program through the National Research Foundation of Korea (NRF) funded by the Ministry of Science, ICT \& Future Planning (Grant No. 2015M1A7A1A01002786). This support is greatly appreciated.

\section{References}

[1] Gould, R.W., O’Neil, T.M. and Malmberg, J.H. (1967) Physical Review Letters, 19, 219-222. http://dx.doi.org/10.1103/physrevlett.19.219

[2] Krall, N.A. and Trivelpiece, A.W. (1974) Principles of Plasma Physics. McGraw-Hill, New York, p. 547. http://dx.doi.org/10.1109/tps.1974.4316834

[3] Malmberg, J.H., Wharton, C.B., Gould, R.W. and O’Neil, T.M. (1968) Physical Review Letters, 20, 95-98. http://dx.doi.org/10.1103/physrevlett.20.95

[4] Sitenko, A.G., Pavlenko, V.N. and Zasenko, V.I. (1975) Physics Letters A, 53, 259-260. 
http://dx.doi.org/10.1016/0375-9601(75)90431-4

[5] Lee, H.J. and Lee, M.-J. (2015) The Open Plasma Physics Journal, 8, 1-7. http://dx.doi.org/10.2174/1876534301508010001

[6] Landau, L. (1946) Journal of Physics, 10, 25-34.

[7] Shivarova, A. and Zhelyazkov, I. (1982) Surface Waves in Gas-Discharge Plasmas. In: Boardman, A.D., Ed., Electromagnetic Surface Modes, Wiley, New York, p. 516.

[8] Vukovic, S. (1986) Surface Waves in Plasmas and Solids. World Scientific, Hong Kong.

[9] Gradov, O.M. and Stenflo, L. (1983) Physics Reports, 94, 111-137. http://dx.doi.org/10.1016/0370-1573(83)90004-2

[10] Lee, H.J. and Lim, Y.K. (2007) Journal of the Korean Physical Society, 50, 1056-1061. http://dx.doi.org/10.3938/jkps.50.1056

[11] Stenflo, L. and Yu, M.Y. (2002) Physics of Plasmas, 9, 5129-5130. http://dx.doi.org/10.1063/1.1521715

\section{Submit or recommend next manuscript to SCIRP and we will provide best service for you:}

Accepting pre-submission inquiries through Email, Facebook, LinkedIn, Twitter, etc.

A wide selection of journals (inclusive of 9 subjects, more than 200 journals)

Providing 24-hour high-quality service

User-friendly online submission system

Fair and swift peer-review system

Efficient typesetting and proofreading procedure

Display of the result of downloads and visits, as well as the number of cited articles

Maximum dissemination of your research work

Submit your manuscript at: http://papersubmission.scirp.org/ 